\title{
DER RHEIN: POETIK DES STROMS \\ ZWISCHEN ELEMENTARISIERUNG UND DOMESTIKATION
}

\begin{abstract}
In the early 19th century, German poets start to conceive of the Rhine river in a significant new way. The water appears as a poetic resource and as a dynamic force of industrial production at the same time. In Hölderlin and Brentano, the Rhine is attributed a twofold quality, whereas the water seems to evoke individuality (kairos) and connectivity (cycle). In poetic terms, the river's domestication by means of hydro-engineering leads to an even more radical concept of elementary savageness, concluding in creations of a so-called new mythology like the famous 'Loreley'.
\end{abstract}

\section{Dualität: Die zweifache Gestalt des Stromes}

Der Rhein ist ein Gebilde in zweifacher Gestalt: (natürliches) Wasser und (kulturelle) Schrift, flüchtiges Ereignis und dauerhafte Institution, eine Zone des Übergangs und der Verbindung. Am Rhein und im Rhein verbindet sich auf besondere Weise das Element Wasser mit den Energien der Sprache und der Literatur.

Wie Lucien Febvre in seinem großartigen Buch Le Rhin: Problèmes d'histoire et d'économie von 1935 (dt. Der Rhein und seine Geschichte, 1994) gezeigt hat, war der Rhein auf längere historische Zeiträume gesehen meist eher ein kultureller Übergangsraum als eine politische Grenze. Der ursprünglich keltische Name des Stromes, Renos, bedeutet schlicht: das fließende Wasser. Der Strom ist ein Inbegriff jenes ewigen Fließens, das formelhaft im 126. Fragment Heraklits ausgedrückt ist. Panta rhei, alles fließt. Verwandlung ist das Grundgesetz der Welt. In der Geschichte Europas bahnte der Rhein eine große Verbindungsschneise durch die Mitte des Kontinents, er ist ein zentrales Landschaftsereignis wie die Alpen, denen er entspringt. Verbindungsschneise heißt: Er verbindet, indem er zugleich trennt. Er verbindet Quelle und Mündung - trennt aber die beiden seitlichen Ufer. Doch auch zwischen links- und rechtsrheinischen Siedlungsgebieten entsteht - bei allen Differenzen - seit den Zeiten der römischen Besiedlung ein intensiver Austausch. Das Grundmuster der römischen Städtegründungen ist bis heute erkennbar geblieben: Es sind im Wesentlichen linksrheinische Stadtanlagen, südlich oder westlich der 'germanischen' Gebiete. Am Rhein und mit Hilfe des Rheins formierte sich eine weiche Grenze, eine Übergangszone zwischen römischer und nichtrömischer Sphäre in Mitteleuropa.

DOI 10.1515/angl.2008.048 
Dann aber, um 1800, macht sich immer stärker eine ambivalente, widersprüchliche Entwicklung bemerkbar. Der Rhein wird zum Vehikel der Abgrenzung, er wird ein ideologisches Argument in der Bildung europäischer Nationalstaaten (vgl. Cepl-Kaufmann/Johanning 2003 und Kiewitz 2003). Zugleich wird sichtbar, dass die verbindende Funktion des Stromes dem wachsenden Bedürfnis des europäischen Verkehrs nicht mehr genügen kann. Der Nutzung des Flusses als transnationalem Verkehrsweg stellten sich gravierende Hemmnisse in den Weg. Am Hochrhein - zwischen Bodensee und Basel - gibt es felsige Klippen und Stufen, die nicht überall entschärft werden konnten; der Rheinfall zu Schaffhausen ist nur das berühmteste dieser unüberwindlichen Hindernisse. Das Wasser hat zuviel an Fallhöhe, zwischen dem Strom und seinem Bett wirken zu große Kräfte. Am Oberrhein, zwischen Basel und Mainz, herrscht ein gegenteiliges Problem. Hier sind es die weiten Umwege, die der Fluss nimmt, die vielen Seitenarme, Schleifen und Windungen, in denen sich das Wasser zu verlieren droht; Umwege, die dem Fluss seine Aufgabe als flüssiges Transportband ebenfalls fast unmöglich machen (vgl. Tümmers 1999).

Die Duplizität des Stromes hat um 1800 mehrere Aspekte, teils geographischer, teils politischer, teils technisch-industrieller Art. Zu Zeiten Goethes und Hölderlins war der Rhein zwischen Deutschland und Frankreich ein Sinnbild gewundener und gefährlicher Wege. Auffallend sind seine großen Schicksalsbiegungen und Richtungsänderungen: im Bündnerland von Ost auf Nord, im Bodensee von Nord nach West, in Basel von West nach Nord, am Mittelrhein zwischen Bingen und Koblenz gar ein veritabler Zickzackkurs. Doch so dramatisch sich diese großen Richtungsänderungen auch ausnehmen - noch problematischer waren um 1800 die vielen kleinen Biegungen und Schleifen. Zwischen Basel und Mainz betrug die tatsächliche Flussstrecke mehr als das Dreifache der Luftlinie. Bemerkenswert ist nun, wann und wie die Gestalt des Flusses als Problem entdeckt wird: Das geschieht zeitgleich in der literarischen Romantik und in der industriellen Wasserbautechnik. Um 1800 beginnen Dichtung und Baukunst zugleich, sich am Rhein zu engagieren - eine erstaunliche Koinzidenz. Das am Ende des 18. Jahrhunderts sich verstärkende literarische Interesse an stürzenden und strömenden Gewässern (etwa in den Stromhymnen Hölderlins oder in den Liedern und Sagen der Rheinromantik) sind auch Ausdruck einer auf neue Weise in die Landschaft ausgreifenden menschlichen Industrie und Ökonomie.

Zwei typische Professionen des 19. Jahrhunderts sind es, die sich dem rauschenden Strom zuwenden: die Figur des poetischen Sammlers und Historikers vergangener Zeiten und der neue, technische Heros des Ingenieurs. Der Karlsruher Wasserbauingenieur Johann Gottfried Tulla wurde um 1817 zum spiritus rector des kanalisierten Geradeauslaufs am Oberrhein; und Friedrich Heinrich von der Hagen wurde mit seiner neudeutschen Edition des Nibelungenliedes 1807 zu einer Gründergestalt des Fachs Germanistik, indem er die Hinwendung zu mittelalterlichen Texten einleitete. 
Während Tulla den Oberrhein bearbeitet, bergen Philologen und phantasievolle Mythenschöpfer das imaginäre Rheingold, heben Brentano, von Arnim und andere die Lieder- und Sagenschätze des Mittelrheins. Auf beiden Wegen geht es darum, die dem Strom innewohnenden Kräfte ökonomisch und kulturell nutzbar zu machen. Aber nicht nur am Rhein sind diese neuen kulturellen Energien zu beobachten: Auch an Donau oder Moldau ertönt das Pathos der großen Flüsse, ihre Stilisierung zu nationalen Allegorien.

Seit Mitte des 18. Jahrhunderts waren die Wasserbaukunst und Landschaftstechnik Mitteleuropas in jene Übergangsphase eingetreten, in der aus der Kolonisierung der Elemente eine systematische industrielle Nutzung werden konnte. Die Kultur des Anlegens von Dämmen und Kanälen hat indes eine viel längere Vorgeschichte. Schon die Antike kannte ingenieurstechnische Eingriffe in das Wasserwesen. "Ein freies Volk auf freiem Grund", proklamiert der späte Faust dort, wo vormals unumschränkt das Wasser herrschte. Fausts Werke sind - im Unterschied zu denjenigen Goethes - industrielle Anlagen auf finanzkapitalistischer Basis; auf die ursprüngliche Akkumulation durch die Einführung des Papiergelds folgen technologische Großphantasien des Kanalbaus und der Eindeichung. Dem Meer und den Sümpfen urbares Land abzutrotzen, wird in der Psychohistorie der Moderne zu einer konstitutiven Metapher für die Arbeit der instrumentellen Vernunft. Im späteren 19. Jahrhundert beschreibt Theodor Storms Schimmelreiter die Fortschritte des Deichbaus und seine Opfer als ein widersprüchliches Wechselspiel von rationaler Kulturtechnik und mythenbesetzter Naturgewalt. Mit vergleichbarer Ambivalenz stellt Grillparzers Armer Spielmann die soziale Frage anhand einer Flussüberschwemmung im Wiener Donaugebiet.

Auch am Rhein findet eine Art Kriegsführung der Ingenieure gegen den Eigensinn des Wassers statt. Die Tragweite der von Tulla unternommenen 'Rektifizierung' war erheblich. Pläne und Diskussionen zur Rheinkorrektur reichen ins 18. Jahrhundert und noch weiter zurück; man hoffte so, wirksam gegen Überschwemmungen und Schwankungen des Wasserstandes vorgehen zu können, vor allem gegen die in den stehenden Sumpfgewässern vermuteten Erreger epidemischer Krankheiten wie Cholera und Malaria. Erst nach dem Wiener Kongress erarbeitete eine französisch-badische Kommission praktikable Vorschläge zur Begradigung des Flussbetts und zur Beseitigung seiner Schleifen und Nebenarme. Die Befestigung des Flusslaufs auf dem Abschnitt zwischen Basel und Worms wurde 1817 begonnen und erst nach gut sechzig Jahren vollendet. Tullas Korrektur verkürzte die Länge des Rheins in diesem Bereich um 80 Kilometer (das sind $23 \%$ der Strecke BaselWorms), indem der Fluss in ein geschlossenes und unverlegbares Bett geleitet wurde (Blackburn 2000, 445). ${ }^{1}$ Damit gewann der Schiffsverkehr eine ver-

1 Zu Tulla vgl. Hübner 1982, 192-96. 
lässliche, im Prinzip ganzjährig nutzbare Wasserstraße. In jenen Abschnitten, die gewunden und langsam dahinströmten, konnte es in strengen Wintern allerdings weiterhin zu Frostblockaden kommen, zu so genannten 'Eisgängen' und katastrophalen Überschwemmungen, wie sie Clemens Brentano in seinem "Moseleisgang-Lied" beschreibt. Überhaupt ist auffällig, dass sich die Literatur um ähnliche hydrographische 'Problemzonen' kümmert, wie sie auch den Ingenieuren zu schaffen machen: den Rheinfall, die Engstellen zwischen Bingen und Koblenz.

\section{Trinität: Der Rhein und SeINe Sprachen}

Die Beziehung zwischen den Energien der Literatur und dem Strömen des Elements gilt auf literarischer Seite sowohl für die lyrische wie für die erzählende Gattung. Auf Seiten des Wassers betreffen sie drei, freilich miteinander verbundene Aspekte: erstens die kinetische Energie des fließenden Wassers, zweitens die ästhetische Dynamik des strömenden und des anbrandenden Wassers und drittens schließlich die kommunikative Dimension des schwingenden und zirkulierenden Elements. Knapp zusammengefasst: Das Wasser fließt, es rauscht, und es trägt; und aus diesen drei Eigenschaften leiten sich seine energetischen Impulse für die Literatur ab.

\subsection{Der fließende Strom}

Die erste Feststellung ist banal, aber unerlässlich: Das flüssige Wasser unterliegt im Zusammenspiel mit den Terrain- und Niveaudifferenzen des festen Erdbodens einer Fließdynamik, die seine Ausbreitungsrichtung linearisiert und es in gebündelter, wenn auch nicht gleichförmiger Bewegung abwärts strömen lässt. Auf diese Weise wird der Fluss - als landschaftliches Phänomen genommen - zu einem Modell der verrinnenden Zeit, die stets von oben (dem früheren Zeitpunkt) nach unten (zum späteren) führt. Darum gilt: "Man kann nicht zweimal in denselben Fluss steigen" (Heraklit, Fr. 91).

Mit dem Geniekult der Stürmer und Dränger gewinnt die heroische Sicht auf den Strom an Anziehungskraft, der ungestüme Wasserlauf wird zum Vorbild einer sich Bahn brechenden freien Persönlichkeit.

Seht den Felsenquell,

Freudehell,

Wie ein Sternenblick

Über Wolken,

Nährten seine Jugend

Gute Geister

Zwischen Klippen im Gebüsch. (Goethe 1998, 316f., Z. 1-7) 
So die Anfangsverse von Goethes 1773 entstandenem Gedicht "Mahomets Gesang”. Flussgedichte, und dieses ist für die Strömungsästhetik um 1800 bahnbrechend, tendieren zur erzählenden Lyrik. Sie sind als Gedichte synchron funktionierende Gebilde und haben doch auch ein sukzessive voranschreitendes Handlungsschema, indem sie den Lauf eines Stromes von seinem Ausgang im hochgelegenen Felsenquell bis zur breit auslaufenden Mündung nachzeichnen. Das Flussbett, ein Lebenslauf.

Friedrich Hölderlins 1802 entstandene "Rheinhymne” entwirft die Biographie einer der wichtigsten Lebensadern Europas. Hier spielen die konkreten geographischen Besonderheiten für die Charakterisierung des Stroms eine entscheidende Rolle. Der Rhein nämlich musste, um er selbst zu werden, "droben von den Brüdern" sich trennen, “dem Tessin und dem Rhodanus” (Hölderlin 1992, 342ff., Z. 34-35). Dass in den Gletschergebieten der Gotthard-Region auf engem Raum zwei große europäische Ströme und mehrere regional bedeutsame Flüsse ihren Ursprung haben, deren Wege sich in gegensätzliche Himmelsrichtungen teilen, dieser Umstand legt es nahe, dem alpinen Quellgebiet ein schicksalhaftes Potential zuzuschreiben.

"Von Treppen des Alpengebirgs" (Z. 4) also beginnt der Held in Hölderlins Rheinhymne seinen Lauf, sobald es ihm gelungen ist, sich aus dem Gestein im Bergesinneren zu befreien, wo "lichtlos er / In den Fesseln sich wälzte" (Z. 29-30). Gebunden in Fels und Eis war die Wasserkraft noch potentielle, im freien Lauf wird sie kinetische Energie. Aus dieser Überlegung wird übrigens auch die besondere Verehrung begreiflich, die den Alpengipfeln in der Naturästhetik seit Mitte des 18. Jahrhunderts und zumal im Rahmen einer Topik des Erhabenen entgegengebracht wurde. Höhe wird, als Ausgangspunkt eines Wasserweges betrachtet, zur Fallhöhe; was ganz oben auf den Schneegipfeln und im Gletschereis sich sammelt, ist ein Reservoir akkumulierter Zeit. In der Elegie "Heimkunft" aus dem Frühjahr 1801 feiert Hölderlin die Gipfel und Gletscher des Hochgebirges als "unermessliche Werkstatt" (1992, 368 ff., Z. 17), wo auf "silbernen Höhen" dauerhaft der "leuchtende Schnee" zu Hause ist (Z. 19-20). Er begreift die Alpen als ein gewaltiges Wasserkraftwerk, das Flüsse ins Land schickt, die halb Europa fruchtbar und prosperierend erhalten.

In den freirhythmischen Gesängen über Rhein und Donau versucht Hölderlin darzustellen, wie sich die Besiedlungsgeschichte Mitteleuropas entlang seiner großen Ströme in steter Wechselwirkung von natürlichen und kulturellen Faktoren entwickeln konnte (vgl. Honold 2000/01). Seine Stromhymnen sind Ausdruck einer neuen, von naturwissenschaftlichen Erkundungen geprägten Sicht auf die landschaftliche Organisation Mitteleuropas als eines elementaren Ökosystems. ${ }^{2}$ Indem Hölderlin die Fluss- und

2 Die Alpen wurden um 1800 als eine Art Laboratorium entdeckt, in dem sich bestimmte Naturphänomene besonders gut erforschen ließen. So brachte der von Goethe geschätzte Horace-Bénédict de Saussure von seinen Bergbesteigun- 
Stromwerdung vor allem am Falle des Rheins ab ovo verfolgt, von den gletschergespeisten Quellflüssen der Schweizer Hochalpen zu den süddeutschen Mittelgebirgen und ihren von Städten und von Weinbergen gesäumten Tälern, rekonstruiert er zugleich das Thema der Soziogenese dieser Landstriche als naturgebundene Siedlungsräume. Das Land, sein Fluss und die an ihm lebenden Menschen haben eine gemeinsame Bildungsgeschichte. Deren wechselseitige Abhängigkeit wird sichtbar, sobald man sich alternative Szenarien vorzustellen versucht, und genau das unternimmt Hölderlins Stromhymne. Was, wenn der Rhein im Engadin tatsächlich in der Verlängerung des Alpenlängstals weiter Richtung Osten geflossen wäre, statt nordwärts abzubiegen? "Anderes hoffte der”, sagt Hölderlin über den jungen Rheinstrom, und dass "Ungeduldig ihn / Nach Asia trieb die königliche Seele. / Doch unverständig ist / Das Wünschen vor dem Schiksaal" (Z. 36-39). Oder wenn das Strombett des Hochrheins bei Basel durch die burgundische Pforte in Richtung Mittelmeer abzweigen würde, wie es ja immerhin für einige zehntausend Jahre der Fall war, ehe der Wasserlauf die Biegung zum Graben der oberrheinischen Tiefebene nehmen konnte?

Der Weg des Stromes ist weder geradlinig und berechenbar, sondern kontingent; wie es mit dem Rhein gemeint ist, verrät die Quelle nicht, obwohl in ihr zu jedem gegebenen Zeitpunkt schon der gesamte folgende Lauf enthalten und entschieden ist. "Ein Rätsel ist Reinentsprungenes" (Z. 46). Was Hölderlin Schicksal nennt - man könnte auch sagen: die Entelechie des Stroms -, setzt sich in der Biographie des Rheins aus drei Faktoren zusammen: aus den Ausgangsbedingungen des Flusses (also Höhenlage, Einzugsgebiet, saisonale Wasserschwankungen etc.), aus den Geländeverhältnissen unterwegs (Verlauf der Gebirgslinien und Gerinne, Aufbau und Dichte des tektonischen Untergrundes) und schließlich aus der Strömungsdynamik selbst.

Dank seines starken Gefälles und der massiven Zuströme hat der Rhein im oberen Verlauf (seiner heroischen Jugend) eine enorme Erosionskraft. Sein kraftvoller Zug durch das tief eingekerbte alpine Flussbett gleicht, so Hölderlin, einer der Großtaten des kleinen Herkules.

Nicht liebt er, wie andere Kinder,

In Wikelbanden zu weinen;

Denn wo die Ufer zuerst

An die Seit ihm schleichen, die krummen,

Und durstig umwindend ihn,

Den Unbedachten, zu ziehn

Und wohl zu behüten begehren

Im eigenen Zahne, lachend

Zerreißt er die Schlangen und stürzt

gen neue Erkenntnisse über den Aufbau der Atmosphäre, die Entstehung und den Verlauf von Gewittern und die meteorologischen Auswirkungen der Elektrizität mit. 
Mit der Beut und wenn in der Eil'

Ein Größerer ihn nicht zähmt,

Ihn wachsen läßt, wie der Bliz, muß er

Die Erde spalten, und wie Bezauberte fliehn

Die Wälder ihm nach und zusammensinkend die Berge. (Z. 62-75)

Beschrieben werden hier die wirksamen Kräfte einer sogenannten rückwärtsschreitenden Erosion. Je näher und je steiler die Ufer dem Fluss kommen, desto mehr Angriffsfläche bieten sie der Wasserkraft, die durch den von solchen Engstellen bewirkten Rückstau noch zusätzlichen Druck gewinnt. Der Größere, der allein die Macht des Rheins bezähmen kann, ist darum kein Bergmassiv, sondern der Bodensee, dessen Sammelbecken das Ende der heroischen Phase darstellt und für relativ konstante Wasserführung auch bei saisonal schwankendem Zustrom sorgt. Befreit von den sich im Seebecken ablagernden Geröll- und Sandmassen, ist der Strom leicht nutzbar für Mühlen und Kraftwerke; sein Wasser hat nun jene charakteristische grüne Farbe, die Goethe beim Besuch des Schaffhauser Rheinfalls hervorhob. Zum Triebrad der Entwicklung Europas prädestinieren den Strom zwei Faktoren: sein Wasservolumen und seine Fließgeschwindigkeit. Bereits der Hochrhein führt, nach dem Zustrom der Aare, eine mittlere Wassermenge von mehr als 1.000 Kubikmetern pro Sekunde - mehr, als die Elbe bei Hamburg oder die Donau bei Passau (Tümmers 1999, 87). Und das enorme Gefälle im Oberlauf ist ein Startkapital, mit dem Industriestandorte wuchern können: am Rheinknie, im Rhein-Neckar-Raum, dem Rhein-Main-Gebiet und in der Region Rhein-Ruhr.

Und schön ists, wie er drauf,

Nachdem er die Berge verlassen,

Stillwandelnd sich im deutschen Lande

Begnüget und das Sehnen stillt

Im guten Geschäffte, wenn er das Land baut

Der Vater Rhein und liebe Kinder nährt

In Städten, die er gegründet. (Z. 83-89)

Als Gründer-'Vater' regt der Rhein den Städtebau und die Niederlassung von Gewerbe und Industrie an, seine Wasserkraft ist ein still wandelnder und verlässlicher Motor guter Geschäfte. Ein Blick auf die Landkarte bestätigt es: Zwischen Basel und Rotterdam sitzt ein Großteil des europäischen Industrie- und Wirtschaftsaufkommens. Wir sind mit Hölderlin weit entfernt, diese Prosperität für naturgegeben zu halten; sie erwächst vielmehr aus der Wechselbeziehung zwischen Elementarkräften und menschlicher Gestaltung; diese beiden zusammen schreiben die Kulturgeschichte der Natur. 


\subsection{Der rauschende Strom}

Der Strom fließt, aber seine Energie zeigt sich nur dort, wo ein Kontakt mit heterogenen Elementen stattfindet, der Übertritt in ein anderes Medium oder einen anderen Aggregatzustand. Wie steht es denn mit der sinnlichen Seite des Wassers, seiner stimulierenden Wirkung auf den Menschen und dessen Streben nach Geld und Glück? Die Triebkräfte des Begehrens, Industrie und Erotik, lassen sich aus hydrographischer Perspektive gemeinsam behandeln. "Ich hört' ein Bächlein rauschen", bekennt der vor Liebessehnsucht rastlose Wanderer in dem Gedicht- und Liederzyklus der Schönen Müllerin. Die energetische Wirkung des Stromes verdankt sich einer Austauschbeziehung des Wassers zu seiner Umgebung; diese Beziehung wird deutlich, wenn 'es' rauscht. Rauschen ist der Resonanzeffekt des Strömens. Nach dem durch das Gefälle des Stromes bewirkten linearen Zeitsinn, den wir bislang betrachtet haben, liegt in der Resonanz des Strömens - im Rauschen also - der zweite und noch vitalere Beitrag des Wassers für die Literatur. Dabei kommt nun ein weiterer paradoxer Umstand ins Spiel: die Tatsache, dass man fließendes Wasser eigentlich gar nicht sehen kann, präziser gesagt: dass man sein Fließen nicht sehen kann.

Idealiter nämlich ist die Fortbewegung selbst unsichtbar - wie übrigens in jedem geschlossenen physikalischen System - und nur an Tempowechseln und Friktionen ablesbar, an Verwirbelungen, Oberflächenstrukturen und Kontaktzonen. Es bilden sich Wellen und Strudel; sie sind als Form stationär, während ihr Grundstoff in beständigem Austausch begriffen ist. Diese wechselnden Modulationsformen des fließenden Wassers sind es, die wir seine Strömung nennen.

In seinem Strömungsverhalten tendiert das Fließgewässer zur Figur des Mäanders; das Wasser bricht bei seinem Geradeauslauf binnen Kurzem in Schlangenlinien aus, es beginnt zu schwingen. Dasselbe Phänomen lässt sich übrigens auch an der Bewegung aufsteigenden Zigarettenrauchs beobachten, der nach kurzer vertikaler Linie in eine ähnliche Schwingungsdynamik mit der unbewegten Umgebungsluft eintritt. Länge und Auslenkungsgrad der Schwingung hängen jeweils von der Mensur des Strömungskanals und der Beschaffenheit des Außenkontakts ab. Im Wasser verstärkt dieser Schwingungseffekt die erodierende Wirkung des Stromes auf asymmetrische Weise. Die naturgeschichtliche Erosion zeigt (und das ist auch für die Poetik eine wichtige Einsicht): Es ist die Bewegung selbst, die sich ihre Form schafft (Kühl 2000, 99). Der Rhein dringt vorwärts und fließt im eigenen Zahne, so Hölderlin. Das gilt selbst heute noch, wo das Flussbett über weite Strecken in Betonmauern gezwängt wurde.

Wo der Strom seine Kraft gegen die felsigen Ufer richtet, beginnt das Wasser zu rauschen und zu brausen. Energie wird frei: Sie ist in diesem Falle nicht ökonomisch nutzbare Energie, sondern Mittel eines poetischen und erotischen Zaubers. Die wohl erfolgreichste Inszenierung des berauschenden und bezaubernden Rheinwassers war Clemens Brentanos romantische 
Erfindung der singenden Zauberin Loreley. Diese verführerische Frau und ihre Poesie werden vom Dichter genau dort platziert, wo Strom und Fels in spektakulärer Weise aufeinander treffen, an jener Engstelle zwischen Bingen und Koblenz, die auch der Schifffahrt und den Ingenieuren Kopfzerbrechen bereitete.

$\mathrm{Zu}$ Bacharach am Rheine

Wohnt eine Zauberin,

Sie war so schön und feine

Und riß viel Herzen hin.

Und brachte viel zu schanden

Der Männer rings umher,

Aus ihren Liebesbanden

War keine Rettung mehr. (Brentano. 535-539, Z. 1-8)

Diese femme fatale von St. Goarshausen hat antike Vorläuferinnen: die Nymphe Echo als trügerisch antwortende Geliebte des Narziss, und die Sirenen, die den Seefahrer Odysseus durch ihr Singen zu verführen versuchten (vgl. Bellmann 1980 und Greiner 2005: 249ff.). Loreley ist die heimliche Herrscherin im mittleren Rheintal, dort, wo der Strom zwischen den Felsen des Schiefergebirges die engste und zugleich tiefste Stelle durchqueren muss, dort, wo Riffe und Stromschnellen die Passage beschwerlich machen und zu langsamer, behutsamster Fahrt zwingen. Man fürchtete eine Zeitlang sogar, Tullas Korrektur könnte den überhängenden Felsen gänzlich unterspülen und ins Wasser stürzen lassen. ${ }^{3}$ Es ist also ein neuralgischer Punkt, an dem sich die Schiffer keinesfalls ablenken lassen dürfen. Nur zu begreiflich, dass Räuber und Wegelagerer den Transporten der Holzflößer genau an dieser Engstelle auflauerten; und ebenfalls leicht begreiflich, dass von dieser Gefahrenstelle die wildesten Gerüchte ausgingen. Wovon aber ging hier eigentlich die Gefahr aus?

Clemens Brentano, nachdem sein Gedicht drei tapfere Ritter die tödliche Klippe hinaufgeschickt hat, sagt es selber in einer Fußnote, die dem LoreleyLied innerhalb des Godwi-Romans beigefügt ist. ${ }^{4}$ Die Loreley, oder als regionaler Ortsname zunächst noch 'Lure-Ley', ${ }^{5}$ ist ein durch die Erosion geformter Echofelsen, der zurückwirft, was immer er zu hören bekommt: sei

3 “Einwänden, daß durch schneller fließende Wasser im Mittelrhein das Wasser sich stauen müsse und der Loreley-Felsen so unterspült werde, begegnete er [Tulla, A. H.] mit den Erfolgen der ersten Durchstiche und Verkürzungen" (Hübner 1982, 193).

4 "Bei Bacharach steht dieser Felsen, Lore Lay genannt, alle vorbeifahrende Schiffer rufen ihn an, und freuen sich des vielfachen Echo's" (Brentano, 539).

5 Der Bestandteil Ley bezeichnet den (Schiefer-)Felsen, das Verb 'luren' oder 'loren' dessen tönende Klangeigenschaft - das Echo des rauschenden Stroms. (Vgl. Daxelmüller 2004, 37). 
es das Rauschen des Flusses, seien es die Melodien vorüberziehender Schiffer.

Wer hat dies Lied gesungen?

Ein Schiffer auf dem Rhein,

Und immer hats geklungen

Von dem drei Ritterstein:

Lore Lay

Lore Lay

Lore Lay

Als wären es meiner drei. (Brentano, 539)

Das Echo ist, wie ein Spiegel, treu und trügerisch zugleich. Es bewahrt die Botschaft und verfälscht ihre Quelle; es vervielfacht die Quelle und vereinfacht ihre Botschaft. Viele Männer müssen unten im Strom sterben, wenn sie es hoch droben vom Felsen tönen hören. Ist da nicht jemand? Ist da nicht eine Frau, die im Abendschein ihre goldenen Locken kämmt?

Ich weiß nicht, was soll es bedeuten,

Daß ich so traurig bin;

Ein Märchen aus uralten Zeiten,

Das kommt mir nicht aus dem Sinn.

Die Luft ist kühl und es dunkelt,

Und ruhig fließt der Rhein;

Der Gipfel des Berges funkelt

Im Abendsonnenschein.

Die schönste Jungfrau sitzet

Dort oben wunderbar,

Ihr gold'nes Geschmeide blitzet,

Sie kämmt ihr goldenes Haar. (Heine, 207)

Längst hat sich Brentanos Frauengestalt verselbständigt, als Heinrich Heine den poetischen Kasus übernimmt. Keineswegs stammt sein "Märchen” aus uralten Zeiten (auch wenn es im Metrum die Nibelungenstrophe bemüht); dass es ihm "nicht aus dem Sinn” geht, liegt an der Omnipräsenz des inzwischen überaus erfolgreich installierten zeitgenössischen Mythos in altem Gewand. Schon Brentanos Gedicht hatte an die Frage gerührt, was wohl zuerst da gewesen sei: die sagenhafte Zauberin oder der Echofelsen. Indem er die Sängerin auf den Drei-Ritterstein führte, gab Brentano dem natürlichen Resonanzphänomen eine erhabene poetische Darstellung. Die singende Felsnymphe und Männer verschlingende Zauberin - das ist die mythologisch 'hohe' und gefährliche Lesart; ihre materialistische Deutung erfolgt dann in der 'niedrigen' Fußnote, die den ablenkenden Klangeffekt damit begründet, dass vorbeifahrende Schiffer hier gerne das Echo auspro- 
bieren. Die Männer erliegen einer Frauengestalt, die sie selbst auf den Felsen projizierten - mit lebhafter Phantasie, begehrlichen Blicken und vom Rauschen erfüllten Ohren.

\subsection{Der zirkulierende Strom}

In der Antike waren Frauen als Quell- und Flussnymphen für die Naturwunder des Fließens zuständig. Die deutsche Romantik lässt weibliche Wasserwesen aus dunklen Tiefen hervorleuchten oder setzt sie amphibienhaft an die Übergangs-Schwelle zwischen Wassersaum und festem Boden. Fouqués Undine, Mörikes schöne Lau, die Melusinen Goethes und Fontanes verkörpern Eigenschaften des Fluidums, in welchem sie zu Hause sind: verwandlungsfähig, schlangengleich züngelnd und schwänzelnd, mal lockend, dann wieder zurückweichend. Dass die Liebe zu diesen ungreifbaren Wesen immer tragisch endet, liegt am Zusammentreffen unvereinbarer Aggregatzustände: Das Flüssige und das Feste bilden keine stabile Gemeinschaft, sondern einen auf ewig spannungsvollen Gegensatz.

Wenn aber flüssiges Strömen und feste Form auf intelligente Weise kombiniert werden, entsteht etwas Drittes: ein stationäres, d.h. auf der Stelle verharrendes Bild des Umschwungs und Kreislaufs: das Rad einer wassergetriebenen Mühle. Ist der Fluss, der tagtäglich an derselben Stelle vorbeizieht, ein und derselbe Fluss, auch wenn sein Wasser ständig wechselt? Ja und nein. Die Figur des Kreislaufs verbindet Dauer und Wandel, sie hat insofern mit der zwiegestaltigen Existenz des Flusses etwas gemein.

"Im Rheingau, wo jetzt Rüdesheim liegt, stand vor undenklichen Zeiten eine einsame Mühle am Rhein, umgeben von einer grünen und blumenreichen Wiese. Auf dieser Mühle wohnte Radlauf, ein junger frommer Müllerbursche" (59). In Clemens Brentanos Märchen vom Rhein ist die 'schöpferische' Erzeugung von Literatur durch strömende Wasserkraft als ein poetologisches Modell ganz und gar wörtlich genommen. Die Vorstellung, dass der Strom das Rad der Poesie treibt, erscheint so plastisch und unmittelbar, wie es nur im Märchenton möglich ist. Wie bei Ovid lautet das Programm: Zurück zu den Anfängen! Die Erzählung holt weit aus, bis in die Anfänge der Siedlungsgeschichte des beschriebenen Landstriches. Das Besondere der Städte am Rheinknick, von Mainz, Bingen, Rüdesheim, und dann auch der Talenge des Mittelrheins, ist nur durch eine genealogische Betrachtungsweise wirklich zu begreifen, durch die Erinnerung an die Elementarbeziehung zwischen Naturkraft und Menschengeschick, die sich am Modell der Mühle herausbildete. Dass jener unvordenkliche Müller am Rhein auf den Namen 'Radlauf' hörte, verrät schon das ganze Geheimnis seiner Berufung. Es ist das fließende Wasser, dem er alles verdankt. Wenn Vater Rhein zu den anderen Bewohnern am Flusse sagt, der Müller Radlauf sei der einzige, mit dem er reden, dem er sich verständlich machen könne, so 
spielt das auf die innige Beziehung an, in der sich Müller und Strom als die beiden Hauptfiguren des Brentanoschen Märchenzyklus befinden.

Der Müller begann und beendete sein Tagwerk mit einer Huldigung an den Rhein; der vorüberziehende Strom war sein Lebensinhalt, seine materielle und kulturelle Existenzgrundlage. Indem der Strom tagaus, tagein die Mühlräder treibt, ermöglicht er Müller Radlauf ein sorgenfreies Auskommen auf reichlich bemessener Grundlage. Dass auf seiner von der Natur begünstigten Wiese die schönsten Blumen blühen, regt den jungen Müller zu kulturellen Dankesopfern an. Nicht aus Elend und Not entspringt hier die Poesie, sondern aus vegetativem Überschuss. "Ehe er [...] schlafen ging, flocht er immer noch einen schönen Blumenkranz und sang dem alten Rhein ein Lied vor, ihm seine Ehrfurcht zu beweisen. Am Schlusse des Lieds warf er dann den Kranz in die Wellen" (59).

Der grüne Kranz scheint hier kein Liebespfand, sondern eher eine Art Götteropfer zu sein; eine Gabe, die durch Verschwendung auf ihre Kosten zu kommen hofft. Der alte treue Rhein, so besagt des Müllers Lied, bleibt wach und regsam, während die Menschenwelt zur Ruhe kommt. Das allmächtige Wasser erlangt in dieser Anrufung religiöse Attribute; die einzelnen Strophen entfalten seine Verehrung als Gottheit und Schicksalsmacht. Manchen lehre der Strom lachen (sagt das Lied), da er Lebenslust verheißt und den Weinbau begünstigt, andere bringe er zum Weinen, da er ihnen die Liebsten entführt und nur ein leeres Echo zurücklässt; für wieder andere heiße es beten, wenn sie vor die Strudel und Abgründe des reißenden Stroms geraten. "Mich aber lehrst du singen", freut sich Müller Radlauf:

Mich aber lehrst du singen,

Wenn dich mein Aug ersieht,

Ein freudseliges Klingen

Mir durch den Busen zieht;

Treib fromm mir meine Mühle, Jetzt scheid ich in der Kühle Und schlummre ein.

Ihr lieben Sterne decket

Mir meinen Vater zu,

Bis mich die Sonne wecket,

Bis dahin mahle du;

Wird's gut, will ich dich preisen,

Dann sing' in höhern Weisen

Ich dir ein Lied.

Nun werf ich dir zum Spiele

Den Kranz in deine Fluth;

Trag' ihn zu seinem Ziele,

Wo dieser Tag auch ruht;

Gut' Nacht! ich muß mich wenden, 
Muß nun mein Singen enden,

Gut Nacht! mein Rhein!

Dieses Loblied auf Vater Rhein setzt die Antriebskraft des Stromes, der die Mühlräder ihren Schwung verdanken, in direkte Analogie zum bewegten Gesang des Müllers; es ist also der Rhein, der das Singen und Sagen in Bewegung setzt.

Die Rahmenhandlung der Rheinmärchen hat, bald nach diesem Idyll, von einem kriegerischen Konflikt zu erzählen, der zwischen den Herrscherhäusern von Mainz und Trier entstanden war. Das symbolische Wappentier der Mainzer ist die Katze, dasjenige von Trier hingegen eine Maus. Im Mainzer Mäusekreuzzug werden (wie in der Geschichte vom Rattenfänger) sämtliche Trierer Mäuse in den Rhein gelockt und ertrinken jämmerlich. Aus Rache lockt der Trierer Gegenspieler Prinz Mausohr gar sämtliche Mainzer Kinder in die Fluten des Rheins. Doch die Kinder ertrinken nicht, sie warten unerlöst und schlafend in der Tiefe des Stromes. Für jede Geschichte, die dem gutmütigen alten Rhein erzählt wird, verspricht er, eines der Kinder wieder freizugeben.

'Kinder gegen Märchen', heißt es nun. Die den Zyklus der Rheinmärchen verbindende Grundidee besteht in der symbolischen Tauschbeziehung zwischen Wasser und Literatur. Die einzelnen Märchen sind Auslösegaben, die dem Rhein dargeboten werden wie der abendliche Blumenkranz des Müllers Radlauf. In dieser Erzählkonstruktion verbinden sich Menschen und Geschichten zu einem Kreislauf. Da aber die Geschichten letztlich aus jenem unversieglichen Vorrat geschöpft sind, den der Strom selber heranführte, gewinnen beide Seiten, die Stadt und der Strom, in diesem Tauschverkehr zurück, was sie je schon besaßen. Was dabei herausspringt? Kein Zugewinn im ökonomischen Sinne zwar, wohl aber die Figur eines geschlossenen Stromkreises. Wie das Wasser, so sind auch die Menschen und ihre Geschichten beständig unterwegs, ein rastloser Umlauf schließt sie alle zusammen.

Der Zyklus der Geschichten, der Blumenkranz des Gedichts - sie erinnern als Figur an die im 19. Jahrhundert immer mächtiger werdende Idee des ökonomischen Kreislaufs, des allgemeinen Austausches von Geld und Waren - und doch ist die 'Ökonomie' des poetischen Kreislaufs, den der Strom antreibt, eine ganz andere. Der grüne Kranz, die dargebotenen Lieder und Märchen sind als Gaben Ausdruck einer kalkulierten, kommunikativen 'Verschwendung'. Lieder sind nichts, was man für sich behält. Zum Kranz runden sie sich erst, wenn ihr Weg stromabwärts die Hörenden erreicht und ihrerseits wieder zu Sängern macht. Oder zu Sängerinnen. In der verschwenderischen Gabe an den Fluss schwingt die Hoffnung mit, an unbekannten Ufern auf liebende Empfänglichkeit zu treffen.

In ihre Sammlung Des Knaben Wunderhorn haben Achim von Arnim und Clemens Brentano einige Lieder aufgenommen, in welchen die Unumkehrbarkeit des Zeitflusses durch die poetische Figur des Zirkels, des Kranzes 
oder Ringleins zauberisch aufgehoben wird. Eines der schönsten davon, mehrfach vertont, unter anderem von Gustav Mahler, ist die folgende Weise:

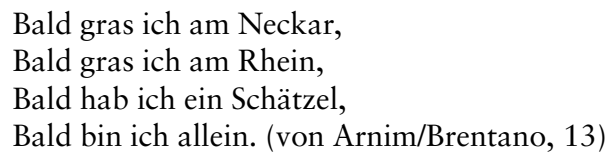

Schlicht und einfach, und doch ein weltkluges Liebeslied. Es spricht drei in ihrem Verhältnis sehr problematische Zustände an; das Alleinsein, die verliebte Zweisamkeit, und das Schwinden der Liebe beziehungsweise den Wechsel der geliebten Person. Wer bald hier, dann wieder dort zu grasen beliebt, muss mit Verlusten rechnen. "Was hilft mir ein Schätzel, / das bei mir nicht bleibt?" Die Liebe zieht den Strom hinab, ihr Wasser bleibt nicht, was es war. Aber sie kommt irgendwann wieder, wofür die Figur des Ringleins zum Zeichen dient.

So soll ich denn grasen

Am Neckar, am Rhein,

So werf ich mein goldiges

Ringlein hinein. (13)

Der große Wurf, das wäre ein Lieben ohne Besitzansprüche. Eines, das sich damit begnügen und trösten könnte, dass alle Flüsse einmal zusammenfinden, und mit ihnen auch jedes Ringlein seinen Adressaten.

\section{Works Cited}

Arnim, Achim von und Ludwig und Clemens Brentano. 1984. Des Knaben Wunderhorn: Alte deutsche Lieder [1805-08]. München: DTV.

Bellmann, Werner. 1980. "Brentanos Lore Lay-Ballade und der antike Echo-Mythos". Clemens Brentano: Beiträge des Kolloquiums im Freien Deutschen Hochstift 1978. Ed. Detlev Lüders. Tübingen: Niemeyer: 1-9.

Blackburn, David. 2000. "Besiegte Natur: Wasser und die Entstehung der modernen deutschen Landschaft". Wasser. Ed. Kunst- und Ausstellungshalle der Bundesrepublik Deutschland, Redaktion Bernd Busch und Larissa Förster. Bonn: Bundeskunsthalle: 440-453.

Brentano, Clemens. 1978. "Zu Bacharach am Rheine" [1802]. Godwi oder Das steinerne Bild der Mutter: Ein verwilderter Roman von Maria. Frankfurter Brentano Ausgabe Bd. 16. Ed. Werner Bellmann. Stuttgart: Kohlhammer.

Cepl-Kaufmann, Gertrude und Antje Johanning. 2003. Mythos Rhein: Zur Kulturgeschichte eines Stromes. Darmstadt: Wissenschaftliche Buchgesellschaft.

Daxelmüller, Christoph. 2004. "Von Zwergen und einem sagenhaften Schatz: Die Loreley und der mittelalterliche Volksglaube”. Die Loreley: Ein Fels im RheinEin deutscher Traum: Ausstellungskatalog. Ed. Mario Kramp und Matthias Schmandt. Mainz: Philipp von Zabern: 37-45. 
Febvre, Lucien. 1994. Der Rhein und seine Geschichte [1935]. Frankfurt/Main: Campus.

Goethe, Johann Wolfgang. 1998. "Mahomets Gedichte 1756-1799”. Sämtliche Werke. Bd. 1, ed. Karl Eibl. Frankfurt/Main: Deutscher Klassiker Verlag.

Greiner, Bernhard. 2005. "Mythische Rede als Echo-Rede: Die Lorelei (Ovid Brentano - Heine)". Mythenkorrekturen: Zu einer paradoxalen Form der Mythenrezeption. Ed. Martin Vöhler und Bernd Seidensticker. Berlin: De Gruyter: 243-261.

Heine, Heinrich. 1975. Sämtliche Werke: Düsseldorfer Ausgabe. Bd. 1: Buch der Lieder [1827]. Ed. Manfred Windfuhr. Hamburg: Hoffmann und Campe.

Hölderlin, Friedrich. 1992. Sämtliche Werke und Briefe. Bd. 1. Ed. Michael Knaupp. München: Hanser.

Honold, Alexander. 2000/01. “ “ Der scheinet aber fast/ Rükwärts zu gehen’: Zur kulturgeographischen Bedeutung der 'Ister'-Hymne”. Hölderlin-Jabrbuch. Tübingen: Mohr: 175-197.

Hübner, Paul. 1982. Der Rhein: Von den Quellen bis zu den Mündungen. München: DTV.

Kiewitz, Susanne. 2003. Poetische Rheinlandschaft: Die Geschichte des Rheins in der Lyrik des 19. Jahrhunderts. Köln: Böhlau.

Kühl, Johannes. 2000. "Strömungen und Wirbel”. Wasser. Ed. Kunst- und Ausstellungshalle der Bundesrepublik Deutschland, Redaktion Bernd Busch und Larissa Förster. Bonn: Bundeskunsthalle: 97-104.

Tümmers, Horst-Johannes. 1999. Der Rhein: Ein europäischer Fluß und seine Geschichte [1994]. 2. Aufl. München: DTV.

BASEL

Alexander Honold 